\title{
On generalized fractional integral inequalities of Ostrowski type
}

\author{
HÜSEYin YildiRIM AND SEdA KILINÇ YILdIRIM
}

\begin{abstract}
We obtain new generalizations of Ostrowski inequality by using generalized Riemann-Liouville fractional integrals. Some special cases are also discussed.
\end{abstract}

\section{Introduction}

Let $f:[a, b] \rightarrow R$ be a function continuous on $[a, b]$ and differentiable in $(a, b)$. If $\left|f^{\prime}(x)\right| \leq M$ for all $x \in(a, b)$, then (see [14])

$$
|f(x)-M(f ; a, b)| \leq \frac{M}{b-a} \frac{(b-x)^{2}+(x-a)^{2}}{2}
$$

for all $x \in[a, b]$, where $M(f ; a, b)=\frac{1}{b-a} \int_{a}^{b} f(x) d x$.

The inequality (1) is well known in the literature as Ostrowski inequality. Over the years, numerous studies have focused on generalizing this inequality, see, for example, $[2-4,6,12,14,21]$ and the references cited therein.

Grüss [5] proved the inequality

$$
|M(f g ; a, b)-M(f ; a, b) M(g ; a, b)| \leq \frac{1}{4}\left(M_{1}-m_{1}\right)\left(M_{2}-m_{2}\right),
$$

where $f$ and $g$ are two integrable function on $[a, b]$ satisfying the conditions $m_{1} \leq f(x) \leq M_{1}$ and $m_{2} \leq g(x) \leq M_{2}$ for all $x \in[a, b]$. The constant $\frac{1}{4}$ is the best possible.

Received January 30, 2021.

2020 Mathematics Subject Classification. 26A33, 26D10, 26D15, 41A55.

Key words and phrases. Fractional integral, Ostrowski inequality, Korkine's identity, Riemann-Liouville fractional integral.

https://doi.org/10.12697/ACUTM.2021.25.09 
Korkine's identity [11] states that if $f$ and $g$ are two integrable function on $[a, b]$, then

$$
\begin{aligned}
& M(f g ; a, b)-M(f ; a, b) M(g ; a, b) \\
& \quad=\frac{1}{2(b-a)^{2}} \int_{a}^{b} \int_{a}^{b}(f(t)-f(s))(g(t)-g(s)) d s d t .
\end{aligned}
$$

Many researches have studied various types of integral inequalities for Riemann-Liouville integrals (see $[7-9,15,18,19,20,22]$ and references therein).

Definition 1 (see [17]). Let $f \in L^{1}[a, b]$. The Riemann-Liouville fractional integrals $J_{a^{+}}^{\alpha} f(x)$ and $J_{b^{-}}^{\alpha} f(x)$ of order $\alpha \geq 0$ are defined, respectively, by

and

$$
J_{a^{+}}^{\alpha} f(x)=\frac{1}{\Gamma(\alpha)} \int_{a}^{x}(x-t)^{\alpha-1} f(t) d t, \quad x>a,
$$

$$
J_{b^{-}}^{\alpha} f(x)=\frac{1}{\Gamma(\alpha)} \int_{x}^{b}(t-x)^{\alpha-1} f(t) d t, \quad x<b .
$$

Here $\Gamma(\alpha)$ is Gamma function and $J_{a^{+}}^{0} f(x)=J_{b^{-}}^{0} f(x)=f(x)$.

$\mathrm{Hu}[7]$ obtains the following generalizations for (1) by using (2), (3), and Riemann-Liouville fractional integrals.

Theorem 1 (see [7]). Let $f$ be differentiable function on $[a, b]$ and let $\left|f^{\prime}(x)\right| \leq M$ for any $x \in[a . b]$. Then the fractional inequality

$$
\left|\frac{(x-a)^{\alpha}+(b-x)^{\alpha}}{\Gamma(\alpha+1)} f(x)-J_{x^{+}}^{\alpha} f(a)-J_{x^{+}}^{\alpha} f(b)\right| \leq M \frac{(x-a)^{\alpha+1}+(b-x)^{\alpha+1}}{\Gamma(\alpha+2)}
$$

holds for any $x \in[a, b]$ and $\alpha \geq 0$.

Theorem 2 (see [7]). Let $f:[a, b] \rightarrow R$ be a differentiable mapping and let $f^{\prime} \in L^{2}[a, b]$. If $f^{\prime}$ is bounded on $[a, b]$ with $m \leq f^{\prime}(x) \leq M$, then we have

$$
\begin{aligned}
& \mid \frac{\alpha f(x)+f(a)}{\Gamma(\alpha)(\alpha+1)}(x-a)^{\alpha-1}-\frac{\alpha}{x-a} J_{x^{-}}^{\alpha} f(a) \\
& \quad+\frac{\alpha f(x)+f(b)}{\Gamma(\alpha)(\alpha+1)}(b-x)^{\alpha-1}-\frac{\alpha}{b-x} J_{x^{+}}^{\alpha} f(b) \mid \\
& \leq \sqrt{\frac{1}{2 \alpha+1}-\frac{1}{(\alpha+1)^{2}} \frac{(x-a)^{\alpha} K_{1}+(b-x)^{\alpha} K_{2}}{\Gamma(\alpha)}} \\
& \leq \sqrt{\frac{1}{2 \alpha+1}-\frac{1}{(\alpha+1)^{2}}} \frac{(x-a)^{\alpha}+(b-x)^{\alpha}}{2 \Gamma(\alpha)}(M-m)
\end{aligned}
$$

for all $x \in[a, b]$ and $\alpha \geq 0$. Here

$$
K_{1}^{2}=M\left({f^{\prime 2}}^{2} ; a, x\right)-M^{2}\left(f^{\prime} ; a, x\right), \quad K_{2}^{2}=M\left({f^{\prime 2}}^{2} ; x, b\right)-M^{2}\left(f^{\prime} ; x, b\right) .
$$


Now we will give definitions of generalized fractional integrals.

Definition 2 (see [9]). The space $L_{p, k}[a, b]$ is defined as

$$
L_{p, k}[a, b]=\left\{f:\|f\|_{p, k}=\left(\int_{a}^{b}|f(t)|^{p} t^{k} d t\right)^{\frac{1}{p}}<\infty\right\}, 1 \leq p<\infty, k \geq 0 .
$$

For $k=0$, the space $L_{p, k}[a, b]$ reduces to the classical space $L^{p}[a, b]$.

Definition 3 (see $[1,9,11]$ ). Let $h(x)$ be an increasing positive monotone function on $[a, b]$ such that $h^{\prime}(x)$ is continuous on $(a, b)$. The space $X_{h}^{p}(a, b)$ $(1 \leq p<\infty)$ is defined as the set of those real-valued Lebesque measurable functions $f$ on $[a, b]$ for which

$$
\|f\|_{X_{h}^{p}}=\left(\int_{a}^{b}|f(t)|^{p} h^{\prime}(t) d t\right)^{\frac{1}{p}}<\infty .
$$

In particular, if we take $h(x)=\frac{x^{k+1}}{k+1}(k \geq 0)$, then the space $X_{h}^{p}(a, b)$ coincides with the space $L_{p, k}[a, b]$. For $h(x)=x$, the space $X_{h}^{p}(a, b)$ coincides with the classical space $L^{p}[a, b]$.

Definition 4 (see $[1,9,11,23]$ ). Let $f \in X_{h}^{p}(a, b)$. The left and right generalized fractional integrals of function $f$ of order $\alpha \geq 0$ are defined, respectively, by

$$
J_{a^{+}, h}^{\alpha} f(x)=\frac{1}{\Gamma(\alpha)} \int_{a}^{x}(h(x)-h(t))^{\alpha-1} h^{\prime}(t) f(t) d t, \quad x>a,
$$

and

$$
J_{b^{-}, h}^{\alpha} f(x)=\frac{1}{\Gamma(\alpha)} \int_{x}^{b}(h(t)-h(x))^{\alpha-1} h^{\prime}(t) f(t) d t, \quad b>x .
$$

Here $\Gamma(\alpha)$ is Gamma function and $J_{a^{+}, h}^{0} f(x)=J_{b^{-}, h}^{0} f(x)=f(x)$.

Remark 1. Letting $h(x)=x$ in (6) and (7), we obtain the equalities in Definition 1.

In this paper we will generalize expressions (1), (3), (4), and (5) by using generalized Riemann-Liouville fractional integrals.

\section{Main results}

Theorem 3. If $f, g \in X_{h}^{p}(a, b)$, then we have, for $\alpha \geq 0$, the identity

$$
\begin{aligned}
& J_{a^{+}, h}^{\alpha}[f(b) g(b)]-\frac{\Gamma(\alpha+1)}{(h(b)-h(a))^{\alpha}} J_{a^{+}, h}^{\alpha}[f(b)] J_{a^{+}, h}^{\alpha}[g(b)] \\
& =\frac{\alpha}{2(h(b)-h(a))^{\alpha} \Gamma(\alpha)} \int_{a}^{b} \int_{a}^{b}(f(t)-f(s))(g(t)-g(s)) \\
& \quad \times(h(b)-h(s))^{\alpha-1}(h(b)-h(t))^{\alpha-1} h^{\prime}(t) h^{\prime}(s) d s d t .
\end{aligned}
$$


Proof. By $(f(t)-f(s))(g(t)-g(s))=f(t) g(t)-f(t) g(s)-f(s) g(t)+$ $f(s) g(s)$ we have

$$
\begin{aligned}
\int_{a}^{b} & \int_{a}^{b}(f(t)-f(s))(g(t)-g(s))(h(b)-h(s))^{\alpha-1} \\
& \times(h(b)-h(t))^{\alpha-1} h^{\prime}(t) h^{\prime}(s) d s d t \\
= & 2\left[\int_{a}^{b}(h(b)-h(s))^{\alpha-1} h^{\prime}(s) d s \int_{a}^{b} f(t) g(t)(h(b)-h(t))^{\alpha-1} h^{\prime}(t) d t\right] \\
& -2\left[\int_{a}^{b} g(s)(h(b)-h(s))^{\alpha-1} h^{\prime}(s) d s \int_{a}^{b} f(t)(h(b)-h(t))^{\alpha-1} h^{\prime}(t) d t\right] \\
= & \frac{2(h(b)-h(a))^{\alpha}}{\alpha} \Gamma(\alpha) J_{a^{+}, h}^{\alpha}[f(b) g(b)]-2 \Gamma^{2}(\alpha) J_{a^{+}, h}^{\alpha}[f(b)] J_{a^{+}, h}^{\alpha}[g(b)] .
\end{aligned}
$$

This completes the proof.

Remark 2. Taking $\alpha=1$ in (8), we obtain the identity

$$
\begin{aligned}
& M_{h}(f g ; a, b)-M_{h}(f ; a, b) M_{h}(g ; a, b) \\
& \quad=\frac{1}{2(h(b)-h(a))^{2}} \int_{a}^{b} \int_{a}^{b}(f(t)-f(s))(g(t)-g(s)) h^{\prime}(t) h^{\prime}(s) d s d t,
\end{aligned}
$$

where $M_{h}(f ; a, b)=\frac{1}{h(b)-h(a)} \int_{a}^{b} f(t) h^{\prime}(t) d t$.

Remark 3. For $h(x)=x$ in (9), we obtain the Korkine's identity (3).

Theorem 4. Let $f$ be a differentiable function on $[a, b]$ and let $\left|f^{\prime}(x)\right| \leq M$ for any $x \in[a, b]$. Then we have, for $\alpha \geq 0$, the generalized fractional inequality

$$
|D(f ; a, b)| \leq \frac{1}{\Gamma(\alpha+2)} M\left[(h(x)-h(a))^{\alpha+1}+(h(b)-h(x))^{\alpha+1}\right],
$$

where

$$
\begin{aligned}
D(f ; a, b)= & \frac{1}{\Gamma(\alpha+1)}\left[(h(x)-h(a))^{\alpha}+(h(b)-h(x))^{\alpha}\right] f(x) \\
& -J_{x^{-}, h}^{\alpha}[f(a)]-J_{x^{+}, h}^{\alpha}[f(b)]-\left[J_{x^{-}, h}^{\alpha+1}\left[\frac{f(a)}{h(a)}\right]+J_{x^{+}, h}^{\alpha+1}\left[\frac{f(b)}{h(b)}\right]\right] .
\end{aligned}
$$

Proof. Using integration by parts for fractional integrals in Definition 5, we have

$$
\begin{aligned}
& J_{x^{-}, h}^{\alpha+1} f^{\prime}(a)=\frac{1}{\Gamma(\alpha+1)}(h(x)-h(a))^{\alpha} f(x)-J_{x^{-}, h}^{\alpha}[f(a)]-J_{x^{-}, h}^{\alpha+1}\left[\frac{f(a)}{h(a)}\right], \\
& J_{x^{+}, h}^{\alpha+1} f^{\prime}(b)=\frac{-1}{\Gamma(\alpha+1)}(h(b)-h(x))^{\alpha} f(x)+J_{x^{+}, h}^{\alpha}[f(b)]+J_{x^{+}, h}^{\alpha+1}\left[\frac{f(b)}{h(b)}\right] .
\end{aligned}
$$


These equalities show that

$$
J_{x^{-}, h}^{\alpha+1} f^{\prime}(a)-J_{x^{+}, h}^{\alpha+1} f^{\prime}(b)=D(f ; a, b) .
$$

Therefore, using (6) and (7), by $\left|f^{\prime}(x)\right| \leq M, x \in[a, b]$, we have

$$
\begin{aligned}
|D(f ; a, b)| & \leq \frac{1}{\Gamma(\alpha+1)} M\left[\int_{a}^{x}(h(t)-h(a))^{\alpha} h^{\prime}(t) d t+\int_{x}^{b}(h(b)-h(t))^{\alpha} h^{\prime}(t) d t\right] \\
& \leq \frac{1}{\Gamma(\alpha+2)} M\left[(h(x)-h(a))^{\alpha+1}+(h(b)-h(x))^{\alpha+1}\right],
\end{aligned}
$$

which completes the proof.

Remark 4. If we take $h(x)=x$ in (10), then we obtain the inequality (4) in Theorem 1.

Remark 5. If $h(x)=0$ and $\alpha=1$, then the inequality (10) reduces to Ostrowski inequality (1).

Theorem 5. Let $f:[a, b] \rightarrow R$ be a differentiable mapping. If $f^{\prime} \in$ $X_{h}^{p}(a, b)$ is bounded on $[a, b]$ with $m \leq f^{\prime}(x) \leq M$, then

$$
\begin{aligned}
& \left|B_{1}(f ; a, b)+B_{2}(f ; a, b)\right| \\
& \leq \sqrt{\frac{1}{2 \alpha+1}-\frac{1}{(\alpha+1)^{2}}} \frac{1}{\Gamma(\alpha)}(h(x)-h(a))^{\alpha} K_{1}+(h(b)-h(x))^{\alpha} K_{2} \\
& \leq \sqrt{\frac{1}{2 \alpha+1}-\frac{1}{(\alpha+1)^{2}}} \frac{1}{\Gamma(\alpha)} \frac{(h(x)-h(a))^{\alpha}+(h(b)-h(x))^{\alpha}}{2}(M-m)
\end{aligned}
$$

for all $x \in[a, b]$ and $\alpha \geq 0$, where

$$
\begin{aligned}
& B_{1}(f ; a, b)= \frac{1}{\Gamma(\alpha)}(h(x)-h(a))^{\alpha-1}\left[f(x)-\frac{1}{(\alpha+1)} \int_{a}^{x} f^{\prime}(t) h^{\prime}(t) d t\right] \\
&-\frac{\alpha}{h(x)-h(a)}\left(J_{x^{-}, h}^{\alpha}[f(a)]+J_{x^{+}, h}^{\alpha+1}\left[\frac{f(a)}{h(a)}\right]\right), \\
& B_{2}(f ; a, b)= \frac{1}{\Gamma(\alpha)}(h(b)-h(x))^{\alpha-1}\left[f(x)+\frac{1}{(\alpha+1)} \int_{x}^{b} f^{\prime}(t) h^{\prime}(t) d t\right] \\
&-\frac{\alpha}{h(b)-h(x)}\left(J_{x^{+}, h}^{\alpha}[f(b)]+{J^{-}, h}_{x^{-}}^{\alpha+1}\left[\frac{f(b)}{h(b)}\right]\right), \\
& K_{1}^{2}=M_{h}\left(f^{\prime^{2}} ; a, x\right)-M_{h}^{2}\left(f^{\prime} ; a, x\right), \quad K_{2}^{2}=M_{h}\left({f^{\prime}}^{2} ; x, b\right)-M_{h}^{2}\left(f^{\prime} ; x, b\right) .
\end{aligned}
$$

Proof. From(6) and (7) we have

$$
\begin{gathered}
\frac{1}{\Gamma(\alpha)(h(x)-h(a))} \int_{a}^{x}(h(t)-h(a))^{\alpha} h^{\prime}(t) f^{\prime}(t) d t=B_{1}(f ; a, b), \\
\frac{-1}{\Gamma(\alpha)(h(b)-h(x))} \int_{x}^{b}(h(b)-h(t))^{\alpha} h^{\prime}(t) f^{\prime}(t) d t=B_{2}(f ; a, b) .
\end{gathered}
$$


Then

$$
\begin{aligned}
B_{1}(f ; a, b)+B_{2} & (f ; a, b)=\frac{1}{\Gamma(\alpha)(h(x)-h(a))} \int_{a}^{x}(h(t)-h(a))^{\alpha} h^{\prime}(t) f^{\prime}(t) d t \\
& -\frac{1}{\Gamma(\alpha)(\alpha+1)}(h(x)-h(a))^{\alpha-1} \int_{a}^{x} f^{\prime}(t) h^{\prime}(t) d t \\
& -\frac{1}{\Gamma(\alpha)(h(b)-h(x))} \int_{x}^{b}(h(b)-h(t))^{\alpha} h^{\prime}(t) f^{\prime}(t) d t \\
& +\frac{1}{\Gamma(\alpha)(\alpha+1)}(h(b)-h(x))^{\alpha-1} \int_{x}^{b} f^{\prime}(t) h^{\prime}(t) d t
\end{aligned}
$$

and by the identity (9) we get

$$
\begin{aligned}
B_{1}(f ; a, b)+B_{2}(f ; a, b) & \\
= & \frac{1}{2 \Gamma(\alpha)(h(x)-h(a))^{2}} \int_{a}^{x} \int_{a}^{x}\left[(h(t)-h(a))^{\alpha}\right. \\
& \left.-(h(s)-h(a))^{\alpha}\right]\left[f^{\prime}(t)-f^{\prime}(s)\right] h^{\prime}(s) h^{\prime}(t) d s d t \\
& +\frac{1}{2 \Gamma(\alpha)(h(b)-h(x))^{2}} \int_{x}^{b} \int_{x}^{b}\left[(h(b)-h(s))^{\alpha}\right. \\
& \left.-(h(b)-h(t))^{\alpha}\right]\left[f^{\prime}(t)-f^{\prime}(s)\right] h^{\prime}(s) h^{\prime}(t) d s d t .
\end{aligned}
$$

Using the Cauchy-Schwarz inequality for double integrals in (12), we obtain that

$$
\begin{aligned}
& \mid \int_{a}^{x} \int_{a}^{x}\left[(h(t)-h(a))^{\alpha}-(h(s)-h(a))^{\alpha}\right]\left[f^{\prime}(t)\right. \\
& \left.\quad-f^{\prime}(s)\right] h^{\prime}(s)^{\frac{1}{2}} h^{\prime}(t)^{\frac{1}{2}} h^{\prime}(s)^{\frac{1}{2}} h^{\prime}(t)^{\frac{1}{2}} d s d t \mid \\
& \leq\left(\int_{a}^{x} \int_{a}^{x}\left[(h(t)-h(a))^{\alpha}-(h(s)-h(a))^{\alpha}\right]^{2} h^{\prime}(s) h^{\prime}(t) d s d t\right)^{\frac{1}{2}} \\
& \quad \times\left(\int_{a}^{x} \int_{a}^{x}\left[f^{\prime}(t)-f^{\prime}(s)\right]^{2} h^{\prime}(s) h^{\prime}(t) d s d t\right)^{\frac{1}{2}} .
\end{aligned}
$$

Since

$$
\begin{aligned}
& \int_{a}^{x} \int_{a}^{x}\left[(h(t)-h(a))^{\alpha}-(h(s)-h(a))^{\alpha}\right]^{2} h^{\prime}(s) h^{\prime}(t) d s d t \\
& \quad=2(h(x)-h(a))^{2 \alpha+2}\left(\frac{1}{2 \alpha+1}-\frac{1}{(\alpha+1)^{2}}\right)
\end{aligned}
$$

and

$$
\int_{a}^{x} \int_{a}^{x}\left[f^{\prime}(t)-f^{\prime}(s)\right]^{2} h^{\prime}(s) h^{\prime}(t) d s d t
$$




$$
=2(h(x)-h(a))^{2}\left[M_{h}\left(f^{\prime^{2}} ; a, x\right)-M_{h}^{2}\left(f^{\prime} ; a, x\right)\right],
$$

by (13) we have

$$
\begin{aligned}
& \mid \frac{1}{2 \Gamma(\alpha)(h(x)-h(a))} \int_{a}^{x} \int_{a}^{x}\left[(h(t)-h(a))^{\alpha}-(h(s)\right. \\
& \left.\quad-h(a))^{\alpha}\right]\left[f^{\prime}(t)-f^{\prime}(s)\right] h^{\prime}(s) h^{\prime}(t) d s d t \mid \\
& \quad \leq \frac{(h(x)-h(a))^{\alpha}}{\Gamma(\alpha)} \sqrt{\frac{1}{2 \alpha+1}-\frac{1}{(\alpha+1)^{2}}}\left[M_{h}\left(f^{\prime^{2}} ; a, x\right)-M_{h}^{2}\left(f^{\prime} ; a, x\right)\right]^{\frac{1}{2}} .
\end{aligned}
$$

Similarly we find that

$$
\begin{aligned}
& \mid \frac{1}{2 \Gamma(\alpha)(h(b)-h(x))} \int_{x}^{b} \int_{x}^{b}\left[(h(b)-h(s))^{\alpha}-(h(b)\right. \\
& \left.\quad-h(t))^{\alpha}\right]\left[f^{\prime}(t)-f^{\prime}(s)\right] h^{\prime}(s) h^{\prime}(t) d s d t \mid \\
& \quad \leq \frac{(h(b)-h(x))^{\alpha}}{\Gamma(\alpha)} \sqrt{\frac{1}{2 \alpha+1}-\frac{1}{(\alpha+1)^{2}}}\left[M_{h}\left(f^{\prime^{2}} ; x, b\right)-M_{h}^{2}\left(f^{\prime} ; x, b\right)\right]^{\frac{1}{2}} .
\end{aligned}
$$

Using (12), (14), and (15), we obtain the first inequality of (11). Moreover, if $m \leq f^{\prime}(x) \leq M$ on $[a, b]$, then by Grüss inequality we get

$$
\begin{aligned}
& 0 \leq \frac{1}{h(x)-h(a)}\left\|f^{\prime}\right\|_{X_{h}^{2}(a, x)}^{2}-\left(M_{h}\left(f^{\prime} ; a, x\right)\right)^{2} \leq \frac{1}{2}(M-m)^{2}, \\
& 0 \leq \frac{1}{h(b)-h(x)}\left\|f^{\prime}\right\|_{X_{h}^{2}(x, b)}^{2}-\left(M_{h}\left(f^{\prime} ; x, b\right)\right)^{2} \leq \frac{1}{2}(M-m)^{2}
\end{aligned}
$$

which proves the last inequality of (11).

Remark 6. If we set $h(x)=x$ in (11), then we obtain the inequality (5) in Theorem 2.

Corollary 1. Under the assumptions of Theorem 5 with $\alpha=1$ the following inequality holds:

$$
\begin{aligned}
& 2 f(x)+\frac{1}{2}\left[\int_{x}^{b} f^{\prime}(t) h^{\prime}(t) d t-\int_{a}^{x} f^{\prime}(t) h^{\prime}(t) d t\right] \\
& \quad-\frac{1}{h(x)-h(a)}\left[J_{x^{-}, h}^{1}[f(a)]+J_{x^{+}, h}^{2}\left[\frac{f(a)}{h(a)}\right]\right]\left[J_{x^{+}, h}^{1}[f(b)]+J_{x^{-}, h}^{2}\left[\frac{f(b)}{h(b)}\right]\right] \\
& \quad \leq \frac{1}{4 \sqrt{3}}(h(b)-h(a))(M-m) .
\end{aligned}
$$


Remark 7. If we set $h(x)=x$ and $\alpha=1$ in (16), then we obtain the inequality $(2.31)$ of $[23]$.

\section{Concluding remarks}

In this study, we presented Ostrowski type generalized inequalities via generalized fractional integrals. It is also shown that the results proved here are a strong generalization of some already published results.

\section{References}

[1] A. Akkurt, M. Esra Yıldırım, and H.Yıldırım, On some integral inequalities for $(k, h)$ Riemann-Lioville fractional integral, New Trends Math. Sci. 4(2) (2016), 138-146.

[2] M. Alomari and M. Darus, Some Ostrowski type inequalities for convex functions with applications, RGMIA 13(1) (2010), Article 3, 13 pp.

[3] S. Belarbi and Z. Dahmani, On some new fractional integral inequalities, J. Inequal. Pure Appl. Math. 10(3) (2009), Article 86, 5 pp.

[4] Z. Dahmani, L. Tabharit, and S. Taf, Some fractional integral inequalities, Nonlinear. Sci. Lett. A 1(2) (2010), 155-160.

[5] S. S. Dragomir, On the Ostrowski's integral inequality for mappings with bounded variation and applications, Math. Inequal. Appl. 1(2) (2001), 59-66.

[6] G. Grüss, Über das Maximum des absoluten Betrages von $\frac{1}{b-a} \int_{a}^{b} f(x) g(x) d x-$ $\frac{1}{(b-a)^{2}} \int_{a}^{b} f(x) d x \int_{a}^{b} g(x) d x$, Math. Z. 39 (1935), 215-226.

[7] Y. Hu, Ostrowski inequality for fractional integrals and related fractional inequalities, TJMM 5 (2013), 85-89.

[8] H. Kalsoom, M. Idrees, A. Kashuri, M. Uzair Awan, and Y.-M. Chu, Some new $\left(p_{1} p_{2}, q_{1} q_{2}\right)$-estimates of Ostrowski-type integral inequalities via n-polynomials s-type convexity, AIMS Math. 5(6) (2020), 7122-7144.

[9] U. N. Katugampola, New approach to a generalized fractional integral, Appl. Math. Comput. 218(3) (2011), 860-865.

[10] M. A. Khan, S. Begum, Y. Khurshid, and Y.-M. Chu, Ostrowski type inequalities involving conformable fractional integrals, J. Inequal. Appl. 2018, Paper No. 70, $14 \mathrm{pp}$.

[11] A. A. Kilbas, H. M. Srivastava, and J. J. Trujillo, Theory and Applications of Fractional Diferential Equations, Elsevier B.V., Amsterdam, 2006.

[12] A. N. Korkine, Sur une therome de M. Tchebychef, C.R. Acad. Sci. Paris 96 (1883), 316-327.

[13] Z. Liu, Some companions of an Ostrowski type inequality and application, J. Inequal. Pure Appl. Math. 10(2) (2009), Article 52, 12 pp.

[14] A. M. Ostrowski, Über die Absolutabweichung einer differentiebaren Function von ihrem integral Mittelwert, Comment. Math. Helv. 10 (1938), 226-227.

[15] B. G. Pachpatte, On a new Ostrowski type inequality in two independent variables, Tamkang J. Math. 32(1) (2001), 45-49.

[16] S. Rashid, M. A. Noor, K. I. Noor, and Y.-M. Chu, Ostrowski type inequalities in the sense of generalized $K$-fractional integral operator for exponentially convex functions, AIMS Math. 5(3) (2020), 2629-2645.

[17] S. G. Samko, A. A. Kilbas, and O. I. Marichev, Fractional Integrals and Derivatives, Theory and Applications, Gordon and Breach, Yverdon, 1993.

[18] M. Z. Sarikaya, On the Ostrowski type integral inequality, Acta Math. Univ. Comenian, 79(1) (2010), 129-134. 
[19] M. Z. Sarikaya, Ostrowski type inequalities involving the right Caputo fractional derivatives belong to $L_{p}$, Facta Univ. Ser. Math. Inform. 27(2) (2012), 191-197.

[20] M. Z. Sarikaya and H. Filiz, Note on the Ostrowski type inequalities for fractional integrals, Vietnam J, Math. 42(2) (2014), 187-190.

[21] M. Z. Sarıkaya and H. Ogunmez, On new inequalities via Riemann-Liouville fractional integration, arXiv:1005.1167v1 [math.CA] 7 May 2010.

[22] N. Ujevic, Sharp inequalities of Simpson type and Ostrowski type, Comput. Math. Appl. 48 (2004), 145-151.

[23] H. Yildirim and Z. Kirtay, Ostrowski inequality for generalized fractional integral and related inequalities, Malaya J. Mat. 2(3) (2014), 322-329.

Department of Mathematics, Faculty of Science and Arts, University of Kahramanmaraş Sütçü İmam,46000, Kahramanmaraş, Turkey

E-mail address: hyildir@ksu.edu.tr

E-mail address: sedakilincmath@gmail.com 